\title{
NUESTRA EXPERIENCIA CON EL CLOMIFENO
}

Dr. Victor Ruiz Velasco ${ }^{1}$

Dr. René Bailón Uriza ${ }^{2}$

Dr. Edmundo Salas ${ }^{3}$

En nuestra primera comunicación "Resultados con el uso de clomifeno" (11), además de presentar nuestros resultados iniciales, hicimos una amplia revisión de la farmacología, propiedades, probables mecanismos de acción y utilización clínica de esta droga, tan utilizada hoy en día para la inducción de la ovulación y por ende la obtención de embarazos en mujeres con problema anovulatorio.

Por ello en esta ocasión, consideramos innecesario repetir estos aspectos y solo recordaremos que el poder antiestrogénico inicial que posee el clomifeno y que ocasiona un aumento en la excreción de gonadotropinas hipofisiarias, por acción probable a través de hipotálamo (7, 8, 11), da por resultado la estimulación ovárica, con la producción de la ovulación y si es posible de un embarazo.

El objeto de esta comunicación, es el reportar nuestros primeros 50 casos completamente terminados, para ver si nuestros resultados iniciales ya reportados persisten, aún en un número mayor de casos y sobre todo, para dar a conocer cuales son los factores que en nuestra experiencia, hay que tomar en cuenta para el mayor éxito en la utilización de este medicamento.

\section{Material y Método}

El estudio comprende 50 pacientes seleccionadas, asistentes al Hospital de Gineco-Obstetricia N 03 del I.M.S. $\mathrm{S}$. Dichas enfermas debían ser aparentemente sanas, sin patología ostensible, especialmente hepática, renal, cardiovascular o nerviosa y portadora de alguno de los siguientes trastornos: Hiperplasia endometrial, anovulación, poliquistosis ovárica, amenorrea, hemorragia uterina disfuncional y galactorrea.

A cada paciente antes de iniciar el tratamiento, se les hizo historia clínica completa y diagnóstico de su alteración ginecoendocrina principal. La mayor parte de ellas fueron sometidas a las siguientes pruebas: pruebas funcionales hepáticas (bilirubinas, cefalín-colesterol, turbidez y floculación del timol, proteínas totales y parciales y transaminasas oxalacética y pirúvica); prueba renal de la depuración de creatinina endógena; general de orina y sedimento;

\footnotetext{
1 Jefe de Servicio. Hospital de Gineco-Obstetricia No. 3 IMSS. México, D. F. Profesor de Gineco-Obstetricia Clínica. U.N.A.M.

2 Gineco-Obstetra de Base. Hospital de Gineco-Obstetricia No 3. IMSS

3 Residente de 3er. año. Hospital de Gineco-Obstetricia No 3. IMSS.
} 
química sanguínea y biometría hemática con recuento de plaquetas. De los tests endocrinos aparte de la citología vaginal cíclica, el estudio seriado de moco cervical, la biopsia de endometrio, y el test de Sims-Huhner; se hicieron además en las primeras 25 pacientes, hormona estimulante del folículo, lodo proteico o captación de lodo 131 y determinación de 17 K. y $17 \mathrm{GH}$.

La mayoría de los tratamientos efectuados fueron administrados en dosis de $50 \mathrm{mg}$. diarios por 5 días, 111 ciclos; y solamente en 13 ciclos más se utilizaron dosis de $100 \mathrm{mg}$. diarios también por 5 días. Estos 124 ciclos de tratamiento tuvieron 5 esquemas de administración: 42 ciclos se administraron del $5^{\circ}$ al $9^{\circ}$ día del ciclo; 6 ciclos se iniciaron en los días 6 y 7; del día 10 al 14, se administraron 49 ciclos; 8 más se iniciaron entre el día 11 y el 16 del ciclo; y por último 19 ciclos más, se dieron a las pacientes durante un período de amenorrea. Ver Tabla IV.

Durante el tratamiento, las pacientes fueron vigiladas semanalmente, con el objeto de investigar sintomatología y la presencia de alguna patología, especialmente ovárica, así como para las tomas de moco cervical y citología vaginal. Se obtenían conjuntamente curvas de temperatura basal y cuando era necesario biopsias de endometrio premenstrual.
En las primeras 25 pacientes, al terminar tres o cuatro ciclos de tratamiento, si antes no se había presentado embarazo, se procedió a efectuar todas las pruebas de laboratorio y gabinete que se practicaron pretratamiento y se valoraron los resultados finales.

Tal y como podemos apreciar en la Tabla I, las 50 pacientes que comprende el estudio correspondieror: principalmente a problemas de Anovulación, Hiperplasia endometrial, Síndrome de ovarios poliquísticos y Amenorrea, contando solo con dos casos de trastornos menstruales con sangrados abundantes y uno de galactorrea.

TABLA I

\section{PATOLOGIAS TRATADAS}

\begin{tabular}{lc}
\hline Tipo & No $^{\mathbf{2}}$ de Casos \\
\hline Anovulación & 19 \\
Hiperplasia endometrial & 16 \\
Síndrome de ovarios poliquísticos & 7 \\
Amenorrea & 5 \\
Trastornos menstruales & \\
$\quad$ (con sangrados anormales) & 2 \\
Galactorrea & 1 \\
TOTAL & -50 \\
\hline
\end{tabular}

\section{Resultados y Comentarios}

Siguiendo la secuencia presentada en el trabajo inicial, analizaremos los resultados en relación primero a la ovulación y posteriormente a los em-

TABLA II

RESULTADOS TOTALES DE ACUERDO A LA PATOLOGIA

\begin{tabular}{lrrrrr}
\hline Tipo & Tipo & Ovulación & Curaciones & Embarazos \\
\hline Anovulación & 19 & 15 & $78,9 \%$ & 7 & 7 \\
Hiperplasia & 16 & 14 & $87,5 \%$ & 9 & 2 \\
Poliquistosis & 7 & 6 & $85,7 \%$ & 3 & 3 \\
Amenorrea & 5 & 3 & $60 \%$ & 1 & 1 \\
Trastornos menstruales & 2 & 1 & $50 \%$ & 1 & 1 \\
Galactorrea & 1 & 1 & $100 \%$ & & 1 \\
TOTAL: & 50 & 40 & $80 \%$ & 21 & 15 \\
\hline
\end{tabular}


barazos, haciendo mención de los efectos indeseables de la droga, así como de las curaciones más o menos permanentes de las patologías tratadas, señalando en la Tabla II los resultados totales.

\section{Ovulación}

El promedio de obtención de ovulación aparente de tan solo un $55 \%$, que para mediados del año 1967, sobre 5.836 pacientes tratadas, reportaron Gleenblat, Mahesh y Pico (3), se ha superado notablemente en los principales estudios, conforme se han ido seleccionando los casos, lo que podemos apreciar en la Tabla III, confeccionada con aquellos trabajos publicados de estudios hechos con grupos de 5 0o más pacientes.

\section{TABLA III}

\begin{tabular}{lcc}
\hline Autor & $\begin{array}{c}\mathbf{N}^{\mathbf{9}} \text { de } \\
\text { pacientes }\end{array}$ & $\begin{array}{c}\mathbf{N}^{\mathbf{9}} \text { de } \\
\text { Ovulación }\end{array}$ \\
\hline Kistner (6) & 50 & 94 \\
Rivo y cols. (9) & 67 & 86 \\
Grant (2) & 63 & 84 \\
Wally cols. (13) & 56 & 80 \\
Whitelaw y cols. (14) & 52 & 79 \\
Roy y cols. (10) & 179 & 78 \\
Greenblatt y cols. (3) & 257 & 77 \\
Kempers y cols. (5) & 50 & 74 \\
Beck y cols. (1) & 129 & 68 \\
Kase y cols. (4) & 81 & 60 \\
Payne y cols. (7) & 63 & 60 \\
Nuestro estudio & 50 & 80 \\
\hline
\end{tabular}

Ya habíamos señalado (11), que lá falta de concordancia en los resultados, así como la diferencia en los éxitos logrados, dependen principalmente de la selección de los casos y sobre todo de la patología que se trate de corregir, lo que hemos confirmado en nuestro estudio, según vemos en la Tabla II en donde se pueden apreciar las diferencias en los porcientos de ovulación, de una patología a la otra.

Esto no quiere decir que la patología en sí sea la determinante de que se logre o no la ovulación, sino del nivel hormonal que acompaña a ese proceso, especialmente en lo que se refiere al nivel estrogénico previo a la administración del medicamento, obteniéndose muchos mejores resultados en los que este nivel es alto, que en aquellos en los cuales la tasa estrogénica es baja. Nuestra experiencia a este respecto es muy demostrativa y la hemos expuesto en la Tabla IV.

Por otra parte, hay otros factores que alteran o modifican los resultados, como son los diferentes esquemas de tratamiento, refiriéndose en ello no solo a las dosis sino también a los días del ciclo en que se administra la droga. Respecto a la dosis, creemos nosotros que esta no es muy determinante, sobre todo cuando se usan dosis bajas, así en la Tabla V damos a conocer nuestros resultados en este aspecto encontrando que no hay diferencia ostensible, cuando se usan dosis de 50 o 100 miligramos. Pero hacemo's notar, que las dosis de $100 \mathrm{mg}$. las hemos utilizado casi siempre en enfermas obesas, y en pacientes con problemas de poliquistosis ovárica, galactorrea o hiperpla-

TABLA IV

RESULTADOS DE ACUERDO AL NIVEL ESTROGENICO

\begin{tabular}{cccc}
\hline Nivel estrogénico previo & Ovulación & Curación & No de Embarazos \\
\hline Alto & $79,6 \%$ & $48,6 \%$ & 9 \\
Bajo & $33,3 \%$ & $10,0 \%$ & 0 \\
\hline
\end{tabular}


sia endometrial, cuando el primer ciclo de $50 \mathrm{mg}$. no dió efecto.

TABLA V

RESULTADOS DE ACUERDO A LA DOSIS

\begin{tabular}{rccc}
\hline & No de Ciclos & $\begin{array}{c}\text { Ovulación } \\
\text { Casos }\end{array}$ & $\begin{array}{c}\text { Ovulación } \\
\text { Ciclos }\end{array}$ \\
\hline $50 \mathrm{Mg}$. & 111 & $80,0 \%$ & $64,5 \%$ \\
$100 \mathrm{Mg}$. & 13 & $71,4 \%$ & $69,2 \%$ \\
\hline
\end{tabular}

En la diferencia en los resultados, evidentemente es más demostrativo el esquema de aplicación en el ciclo. En la Tabla VI, podemos apreciar que cuando la medicación se inicia en los primeros días del ciclo 5, 6 y 7, el tanto por ciento de ovulación es mucho mayor que cuando se inicia más allá del día 10 y sobre todo cuando se da durante un período de amenorrea, en cuyo caso se obtienen tan sólo un $10 \%$ de ovulaciones contra más del $80 \%$ cuando se da en los primeros días. Esto es en lo único que ha cambiado nuestra manera de pensar conforme ha aumentado nuestra experiencia, ya que inicialmente pensábamos que cuando se daba la droga alrededor del día 10 los resultados serían mejores porque la tasa de estrógenos en el ciclo eran mayores, logrando en aquella ocasión un $63 \%$ de ovulaciones, que indudablemente hemos aumentado hasta por arriba del $80 \%$ en la actualidad.

TABLA VI

RESULTADOS DE ACUERDO AL DIA DE INICIO DE LA MEDICACION DENTRO DEL. CICLO

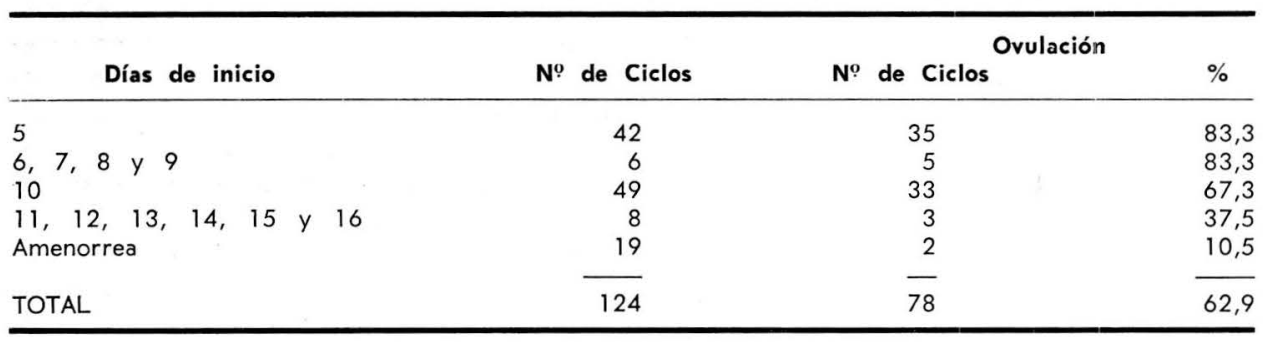

Esta diferente forma de administrar el medicamento durante el ciclo menstrual, ocasiona igualmente cambios en la fecha de la ovulación y por consecuente cambios en la longitud del ciclo, que hay que tomar en cuenta no solamente para no pensar en embarazos iniciales falsos, sino también para indicar a la pareja las fechas más adecuadas para el coito (12). En nuestros casos, hemos visto que mientras más tardíamente se empiece la medicación durante el ciclo, más posterior es la ovulación y por lo tanto más largo es el ciclo. Tabla VII.
TABLA VII

DURACION DEL CICLO EN RELACION A LA FECHA DE INICIO DEL TRATAMIENTO

\begin{tabular}{lc}
\hline Fecha de inicio & Promedio de duración \\
\hline Día 5 & 30.8 días \\
Día 10 & 34.4 días \\
Días 12 a 16 & 41 días \\
\hline
\end{tabular}

\section{Embarazos}

Desgraciadamente la obtención de ovulación no ha correspondido en la práctica al logro de embarazo, lo que se puede explicar porque nuestros métodos de estudio de la pareja es- 
téril son deficientes y a parte del factor anovulatorio pueden existir otros desconocidos y que al persistir impiden el embarazo; o porque nuestros métodos de detección de la ovulación no son tan acertados como pensamos, obteniéndose en realidad menos ovulaciones de las que se cree. Pudiendo quedar como una tercera posibilidad, la de que aunque se lograse el desprendimiento del óvulo, éste no tuviese todas sus facultades para poder ser fecundado. Cualquiera que sea la causa, el hecho real es el de que solamente alrededor del $25 \%$ de las pacientes se hacen embarazadas (3).

Por nuestra parte hemos obtenido 15 embarazos, que si eliminamos 7 pacientes tratadas por hiperplasia $y$ que por su edad no podían embarazarse, nos dá un índice de $35 \%$ de embarazos, algo menor que el $42,1 \%$ inicial que tuvimos (11).

De ellos abortaron el 20\%, Tabla VIII, señalando que dos de los abortos fueron en la misma enferma, una paciente con 17 años de esterilidad primaria y con 38 años de edad, tratada por hiperplasia endometrial adenomatosa. Esta incidencia de aborto, es algo menor que la del promedio general, de 1 por cada 4 pacientes. Además hay que resaltar el hecho de que los 12 embarazos restantes, evolucinaron satisfactoriamente a término, sin que ninguno de ellos fuera múltiple. Esta es otra de las ventajas de utilizar dosis bajas.

\section{TABLA VIII}

E M B A R A Z O S



Por otra parte, ya anotábamos en las Tablas II y IV como el número de embarazos es diferente según la patología tratada y en especial según el nivel estrogénico reinante.

\section{Curaciones}

En nuestra publicación preliminar señalábamos, que según nuestro criterio deberían de empezar a darse los resultados en forma de curaciones y no solo como resultados transitorios o paliativos al lograr una ovulación y así señalábamos que considerábamos los casos de hiperplasia endometrial como curados, cuando el endometrio posterior a la terminación del tratamiento (por lo general a los 3 meses) no muestra signos de dicha hiperplasia. Los casos de ano- vulación se anotaron como éxito solo cuando se logró la concepción. Cuando había el diagnóstico de ovarios poliquísticos, se tomó como curación cuando hubo gestación o desaparecieron el crecimiento ovárico, la amenorrea, la anovulación, la obesidad, etc. Para la amenorrea es imás fácil, ya que es necesario que desaparezca, produciéndose ciclos menstruales ovulatorios, posterior a la administración del clomifeno. Una cosé similar tomamos en cuenta para la hemorragia disfuncional, la cual debe desaparecer durante meses después del tratamiento, teniendo en su lugar ciclos menstruales ovulatorios en forma regular. Por último, la galactorrea se dio por curada cuando desapareció el síntoma. 
Tomando en cuenta lo anterior creemos que 21 de nuestros casos se han curado, dependiendo dicha curación al igual que la ovulación y los embarazos, del tipo de patología tratada y del nivel estrogénico previo. Tablas II y IV.

\section{Tolerancia}

La gran cantidad de efectos colaterales que en un principio se produjeron con la droga importantes por su frecuencia hasta en un $50 \%$ y su gravedad (ruptura de quistes ováricos, trastornos de visión, pérdida del cabello, etc.) $(2,3,11,13)$ y que fueron ocasionados por las enormes dosis que se utilizaron en las investigaciones iniciales, prácticamente han desaparecido en los esquemas modernos de tratamiento.
TABLA IX

\section{EFECTOS INDESEABLES ENCONTRADOS}

Inflamación

Dolor en hemi abdomen inferior

Náuseas y vómito

Cefalea

Mareos

Nerviosidad

Somnolencia

Crecimiento ovárico

Con las dosis bajas como las que hemos utilizado nosotros, sólo se presentan en forma transitoria $y$ nunca con carácter de importancia. Los síntomas que se han encontrado en nuestras pacientes, están anotados en la Tabla $\mathrm{N}$ : IX y se presentaron solo en el $28 \%$ de los casos, es decir más o menos en una de cada cuatro pacientes. Tabla $X$.

TABLA $X$

TOLERANCIA DE LA DROGA

\begin{tabular}{|c|c|c|c|c|c|c|}
\hline $\mathbf{N}^{0}$ & $\begin{array}{l}\text { de pacientes } \\
\text { tratadas }\end{array}$ & $\begin{array}{l}N^{0} \text { de pacientes } \\
\sin \text { síntomas }\end{array}$ & $\%$ & $\begin{array}{l}\text { Nọ de ciclos } \\
\text { administrados }\end{array}$ & $\begin{array}{l}\mathrm{N}^{0} \text { de Ciclos } \\
\text { sin síntomas }\end{array}$ & $\%$ \\
\hline & 50 & 36 & 72 & 124 & 89 & 72 \\
\hline
\end{tabular}

Por último, ya señalamos en nuestro estudio inicial, que de las exhaustivas pruebas de laboratorio practicadas a las primeras pacientes, no encontramos ninguna alteración notable en el control, únicamente que en 5 de los 28 casos en que fueron llevadas a cabo pruebas hepáticas, se produjo una pequeña disminución de las proteínas totales y de la relación $A / G$, así como una elevación de las transaminasas oxalacéticas y pirúvicas, en 4 de ellos.

\section{Resumen y Conclusiones}

Se estudian 50 pacientes seleccionadas asistentes al Hospital de Gineco-Obstetricia No 3 del I.M.S.S., so- metidas a tratamiento con citrato de clomifeno. La mayoría de las pacientes fueron tratadas por anovulación, hiperplasia endometrial, ovarios poliquísticos y amenorrea.

En 111 ciclos se administraron 50 mg. por 5 días y solo en 13 se administró una dosis de $100 \mathrm{mg}$. Se utilizaron varios esquemas de tratamiento: 49 ciclos empezando el día 10 del ciclo menstrual; 42 empezando el 5 : día; 19 durante un período de amenorrea; 8 entre el 11 y el 16; y 6 más empezando los días 6 y 7 del ciclo.

Creemos que la dosis de $50 \mathrm{mg}$. es suficiente. Administramos $100 \mathrm{mg}$. 
solo en enfermas muy obesas y en enfermas con poliquistosis ovárica, hiperplasia o galactorrea, que no responden a una dosis inicial de 50 mg.

En el $80 \%$ de los casos se obtuvo ovulación. Lográndose una mayor incidencia en los casos tratados por Anovulación, Hiperplasia y por Ovarios poliquísticos.

Es determinante para conseguir éxito el nivel estrogénico existente previo al tratamiento, teniendo pocos éxitos cuando dicho nivel es bajo.

Igualmente, se obtienen mejores resultados cuando el medicamento se da en los primeros días del ciclo.

Obtuvimos un 35\% de embarazos en las pacientes que estaban en posibilidad de hacerlo. El $20 \%$ de estos embarazos terminaron en aborto $y$ el resto tuvo fetos normales de término. No tuvimos ningún embarazo múltiple.

Una de cada cuatro pacientes presentó efectos colaterales por la toma de la droga, todos ellos transitorios y de poca importancia.

Por lo anterior concluímos que la administración del clomifeno, debe hacerse en pacientes seleccionadas, sin patología hepática o metabólica, ni cuando existen quistes ováricos o sospecha de neoplasia, pacientes que tengan ovarios e hipófisis en posibilidad de ser estimulados y que presenten una tasa estrogénica buena. La administración del medicamento debe hacerse los primeros días del ciclo y a dosis bajas de 50 miligramos, que logrando los mismos resultados, evitan la aparición de efectos colaterales desagradables y de embarazos múltiples.

\section{REFERENCIAS}

1 BECK, P.; GRAYZEL, E. F.; YOUNG, I. S. y KUPPERMAN, H. S.: Obst. \& Gynec. 27: $54,1966$.

2 GRANT, A.: Australian \& New Zeland J. Obst. \& Gynaec. 6: 327, 1966.

3 GREENBLATH, R. B.; MAHESH, V. B. $y$ PICO I.: Stimulation of Ovulation in the human with Clomiphene Citrate. Fifth World Congress of Gynecology and Obstetrics. Sydney. 1967.

4 KASE, N., NROUEH, A. y OLSON, L. E. Am. J. Obst. \& Gyriec. 98: 1037, 1967.

5 KEMPERS R. D., DECKER D. G. y LEE R. A.: Obstet y Gynec. 30: 669, 1968.

6 KISTNER, R. W.: Am. J. Obst. \& Gynec. 92: 380. 1965.

7 PAYNE, S. Y KAROW, W. G.: West. J. Surg. 71: 262, 1963.

8 PLUNKETT E. R. y YUZPE A.: Am. J. Obst. \& Gynec. 100: 506, 1968.

9 RIVO, E., y ROCK, J.: Pacific Med. \& Surg. 73: 413, 1965.

10 ROY, S.; GREENBLATH; MAHESH, V., Y JUNGCK, E. C.: Fertil. \& Steril. 14: 575. 1963.

11 RUIZ VELASCO V., BAILON R. Y SALAS E. : Ginec. Obstet. Mex. 23: 643, 1968.

12 RUIZ VELASCO V., MATUTE M. Y BAILON R.: Estudios sobre esterilidad. En prensa.

13 WALL, J. A. ; FRANKIN, R. R.; KAUFMAN, R. H., y KAPLAN, A. L.: Am. J. Obst. \& Gynec. 93: 842, 1962.

14 WHITELAW, M. J.; GRAMS, L. R. ; Y STAMM, W. J.: Obst. \& Gynec. 90: 355, 1964. 